illness play an important role in the context. Leaders serve as role models and therefore tend to have a huge impact on the pressure perceived by employees. Moreover, support from management, leadership qualities as well as leadership style tend to be an issue. Correlations often were no longer significant when other variables such as health problems, demographic variables or organisational factors were incorporated.

Discussion Results from quantitative and qualitative studies suggest that there is a correlation between leadership and presenteeism. However, results are not conclusive. Moreover, significant correlations disappeared when multivariate analyses were undertaken. Thus, results have to be interpreted with care.

\section{NIGHT WORK AND COMMON MENTAL DISORDERS: ANALYSING OBSERVATIONAL DATA AS A NON- RANDOMISED PSEUDO TRIAL}

${ }^{1}$ MS Beltagy*, 1J Pentti, ${ }^{2} \mathrm{~J}$ Vahtera, ${ }^{1,3,4} \mathrm{M}$ Kivimäki. 'Department of Public Health, University of Helsinki, Helsinki, Finland; '2Department of Public Health, University of Turku, Turku, Finland; ${ }^{3}$ Department of Epidemiology and Public Health, University College London, London, UK; ${ }^{4}$ Finnish Institute of Occupational Health, Helsinki, Finland

\subsection{6/oemed-2018-ICOHabstracts. 1730}

Introduction This paper investigates the relationship between working regularly in night shifts for long periods and common mental disorders, including depression, anxiety disorders, severe stress, adjustment disorders, neurotic disorders and insomnia. The investigation of this association can help position intervention policies concerned with regulations of work schedule and workers' mental health.

Methods A non-randomised pseudo trial was conducted on two populations of social and healthcare employees (the Finnish Public Sector Study) for a duration of eight years. Study one tested the effect of moving from continuous day work to work in night shifts on the development of common mental disorders for 30019 employees and study two examined the recovery rate from mental disorders when 944 night workers with common mental disorders changed their working schedule to day shifts. Data were analysed using logistic regression with GEE.

Result Study one showed a 3\% higher odds of acquiring common mental disorders among night workers compared to day workers; (odds ratio 1.03, 95\% CI: 0.82 to 1.30 ) whereas study two showed a 99\% higher odds of recovery from common mental disorders among night workers who changed their work schedule to day shifts compared to night workers who continued to work night shifts (odds ratio 1.99, 95\% CI: 1.20 to 3.28). Both results were adjusted for several covariates.

Discussion Our paper introduces a novel approach to analyse observational repeat data on night work which is closer to randomised controlled trials than conventional epidemiologic analyses. The results from study one could not support a causal association between night work and the development of common mental disorders. However, study two shows increased recovery rates from common mental disorders when night workers change their work schedule back to day work. These findings suggest that change in work schedule may have a role in the prognosis of common mental disorders.
880 HEALTHY ENTERPRISE STANDARD (HES) EVALUATION: IMPACT ON ADVERSE PSYCHOSOCIAL WORK FACTORS AND PSYCHOLOGICAL DISTRESS

${ }^{1,2} \mathrm{M}$-C Letellier*, ${ }^{2} \mathrm{C}$ Duchaine, ${ }^{3} \mathrm{M}$ Vézina, ${ }^{1,2} \mathrm{~K}$ Aubé, ${ }^{3} \mathrm{M}-\mathrm{M}$ Mantha-Bélisle, ${ }^{4} \mathrm{H}$ SultanTaïeb, ${ }^{5} \mathrm{~F}$ St-Hilaire, ${ }^{1,2} \mathrm{C}$ Brisson. ${ }^{1}$ Faculty of Medicine, Laval University, Quebec City, Canada; ${ }^{2} \mathrm{CHU}$ de Québec Research Centre, Population Health and Optimal Health Practices Unit, Quebec City, Canada; Institut national de santé publique du Québec, Quebec City, Canada; ${ }^{4}$ Université du Québec à Montréal, Montreal, Canada; ${ }^{5}$ Management School, University of Sherbrooke, Sherbrooke, Canada

\subsection{6/oemed-2018-ICOHabstracts. 1731}

Introduction Adverse psychosocial work factors are recognised as a significant source of psychological distress. The Healthy Enterprise Standard (HES) targets four intervention areas: Lifestyle, Work-life balance, Workplace environment and Management practices; this latter area referring to interventions aimed at improving the psychosocial work environment. The aim of this study was to evaluate the impact of implementing the HES on exposure to adverse psychosocial work factors and the prevalence of psychological distress among ten Quebec organisations.

Methods This was an intervention study with a before-after design derived from secondary data. Organisations adopted the standard of their own initiative and were responsible for implementing interventions. All active employees were solicited to participate before $(\mathrm{T} 1=2849)$ and $24-38$ months $(\mathrm{T} 2=2560)$ following the standard's implementation. At both time points, participants completed a questionnaire. Psychosocial work factors were assessed with the validated demandcontrol-support and effort-reward imbalance models. Psychological distress was assessed with the validated K6. Intervention exposure was measured by questionnaire and complemented by qualitative analyses.

Result The prevalence of low social support at work and high psychological distress decreased among all participants between T1 and T2. In organisations that were less exposed to interventions in the Management practices area, an increase in the prevalence of several adverse psychosocial work factors was observed while a decrease in the prevalence of these adverse factors was observed in organisations more exposed to Management practices interventions. Moreover, a more important decrease in high psychological distress was observed in organisations more exposed compared to those less exposed to interventions in this area.

Discussion These results suggest that organisational psychosocial interventions implemented in the context of this standard may be effective in reducing both adverse psychosocial work factors as well as high psychological distress and may therefore be an effective means to improve the mental health of workers.

\section{THE INFLUENCE OF WORKPLACE SOCIAL CAPITAL ON HBA1C LEVELS AMONG JAPANESE WORKERS: A 2-YEAR PROSPECTIVE COHORT STUDY (J-HOPE)}

${ }^{1}$ Hisashi Eguchi, ${ }^{1}$ Akizumi Tsutsumi, ${ }^{1}$ Akiomi Inoue, ${ }^{2}$ Ichiro Kawachi. 'Department of Public Health, Kitasato University School of Medicine, Sagamihara, Japan; ${ }^{2}$ Department of Social and Behavioural Sciences, Harvard T.H. Chan School of Public Health, Boston, MA, USA

10.1136/oemed-2018-ICOHabstracts. 1732 